\title{
Understanding DSM-5. Advice for South African practitioners
}

What is a clinical case? Which were the criteria for putting people in the Robben Island Lunatic Asylum in 1847, the time when insanity was medicalized and moral management instituted? Such riddles continue to challenge, although we are doing a good job with defining clinically useful diagnoses compared to the uncertainties surrounding many somatic diagnoses such as appendicitis, thyroid disease and neuropathic pain.

Over the years, careful case descriptions were crafted by icons that remain valid and recognizable to the psychiatric community (Table I).

Table l: Brief history of classic diagnoses and classifications

81-138 Aretaeus: bipolar disease

1746 Carl von Linné: Morbi mentales: Dementia, Mania, Vesania, Delirium, Melancholia

1769 William Cullen: neurosis

1845 Wilhelm Griesinger: Pathologie und Therapie der psychischen Krankheiten

1849 Magnus Huss: Alcoholismus chronicus

1860 Florence Nightingale: Hospital morbidity tabulation

1873 Armand Trosseau/Gilles de la Tourette: Tourette syndrome

1890 Gilles de la Tourette: Anorexia nervosa

1891 Arnold Pick/Emil Kraepelin: Dementia praecox

1908 Eugen Bleuler: Schizophrenia

1901 Paul Hartenberg: La Timidité (social anxiety disorder)

1844 APA Classification for institutionalized patients

1947 U.S. National Institute of Mental Health

1947 Erik Essen-Möller: Multiaxial system proposal

1948 WHO: Classification of diseases and causes of death (ICD)

1952 DSM-I

1961 Thomas Szasz: The Myth of Mental IIIness

1968 DSM-II

1980 DSM-III

1987 DSM-III-R

1994 DSM-IV

2000 DSM-IV-TR

Diagnoses were put to a test in WWII. U.S. generals were upset as numerous recruits were disqualified for combat by psychiatrists on capricious grounds, so the NIMH was established in 1947. But diagnostic confusion prevailed, since you need a scientific method. Such a method was first proposed for schizophrenia by Eli Robins and Sam Guze who in their seminal paper described a method for achieving diagnostic validity consisting of five phases: clinical description, laboratory study, exclusion of other disorders, follow-up study, and familial study. ${ }^{1}$ Their studious and unassuming approach to making diagnoses became the compass for the multiaxial and atheoretical DSM-III in 1980. Its market success inspired subsequent editions built on expert consensus.

On 18 May 2013 DSM-5 was released at the APA meeting in San Francisco. ${ }^{2}$

A number of key issues need to be mentioned:

- The scope of DSM-5 was to build on 20 years of research since DSM-IV and to reorganize it in a

neurodevelopmental perspective. This was undertaken through appointing 130 unbiased experts to task forces, supported by 400 consultants and 13000 signed web comments, as well as testing the products in field trials in 11 academic centers.

- Diagnostic criteria were only to be modified if there were new data. Gender differences were to be elucidated.

- Continuum became a lead term to rate the severity of a condition by assessing the degree of impairment in functioning it caused.

- The multiaxial system was substituted for by a principal diagnosis and secondary diagnoses and collaboration established with the WHO in Geneva, to harmonize with ICD-11 that is to be released in 2015.

- The WHO Disability Assessment Schedule (WHODAS $2.0)^{3,4}$ is the generic tool recommended by DSM-5 for assessing the amount of impairment, as rated by the patient, by proxy or by a clinician. Translations are available or ongoing in 31 languages.

- A Cultural Formulation Interview (CFI) is included in DSM-5 to capture the patient's cultural identity, illness attributions, vulnerability and resilience, and how culture and ethnicity affect the interaction with a clinician. ${ }^{5}$

- The preamble to DSM-5 includes a definition of a mental disorder, and what constitutes a clinical case; it defines the utility of DSM-5 as a diagnostic aid (as is the ECG for the cardiologist) and emphasises that there is no substitute for good clinical judgement:

- "... a syndrome characterized by clinically significant disturbance in an individual's cognition, emotion regulation, or behavior that reflects a dysfunction in the psychological, biological, or developmental processes underlying mental functioning."

"The diagnosis of a mental disorder should have clinical utility."

'"The fact that some individuals do not show all symptoms indicative of a diagnosis should not be used to justify limiting their access to appropriate care." 
The major changes in DSM-5 compared to DSM-IV are the following:

1) Autism spectrum disorder includes Asperger and pervasive developmental disorder.

2) Bipolar and depressive disorders have been scrutinized and streamlined, dealing properly with grief as an independent and associated companion to depressive disorders. Grief is indeed qualitatively different from depression.

3) Continuum is the new approach to substance use disorders; doing away with the ambiguous terms dependence and abuse. Tolerance and withdrawal occur with many prescribed psychoactive medications, confounding the concept of addiction and dependence. For example, alcohol use disorder is now specified as mild (F10.10), moderate (F10.20) or severe (F10.20), the latter two based on having at least 4 of 11 symptoms such as craving, tolerance, withdrawal, unsuccessful efforts to cut down, and failure to fulfill major role obligations.

4) The organic brain diseases chapter in DSM-IV has been revised. Dementias are now specified into major and mild neurocognitive disorders, building on the investments into dementia research in recent years.

5) The categorical Personality disorders remain virtually as they were in DSM-IV, although a lot of effort was put into replacing them with the continuum approach used in personality research (5-factor model).

In perusing the manual, I note that Catatonia has its own section based on up to 12 psychomotor features. I am surprised to see enuresis and encopresis as psychiatric diagnoses; they belong better in neurology. It has been rumoured that ICD-1 1 will move sexual dysfunctions (early and late orgasms, erectile dysfunction and low libido) out of the psychiatry chapter into a sex medicine chapter. That makes sense to me. DSM-5 requires that for a psychiatric diagnosis of a paraphilic disorder, there must be clinically significant distress for the individual.

An interesting chapter is that on Somatic Symptom and Related Disorders. It replaces the DSM-IV chapter on somatoform disorders, and it concerns those patients seen in primary care with a focus on prominent somatic symptomatology. In DSM-IV, these diagnoses were made if there was no identifiable somatic disease. Now, the criteria are sharpened, building primarily on distress and dysfunction with our without concurrent somatic disease: " It is not appropriate to give an individual a mental disorder diagnosis solely because a medical cause cannot be demonstrated". So 3 out of 4 patients with hypochondriasis are now subsumed under the new diagnosis Somatic Symptom Disorder because of excessive worry about illness, repeated bodily checking for abnormalities and seeing many doctors. This is considered to be a diagnosis seen in global primary care, albeit the influence of culture and ethnicity is recognized. To have an Illness Anxiety Disorder there is a cognitive preoccupation with having or acquiring a serious illness without a focus on somatic symptoms. Conversion Disorder is diagnosed when the symptoms are incompatible with underlying organic function. There is also Factitious Disorder, including what we also know as Münchhausen syndrome.

Educators should now adjust their curriculum accordingly. As a teacher I will make these adjustments because the continuum approach is useful. Clinical psychiatry will be improved by applying the thinking in DSM-5; it makes sense to me as a clinician.

I hope that the WHO DAS 2.0 will be put to use as well as the CFI. DSM-5 is not the final answer and it is not static. There will be subsequent improvements as new research data emerge to sharpen the phenotypes.

\section{Allgulander}

Karolinska Institutet, Stockholm, Sweden \& University of Cape Town, Cape Town, South Africa

\section{References}

1. Robins E, Guze SB. Establishment of diagnostic validity in in psychiatric illness: Its application to schizophrenia. Am J Psychiatry 1970:126:107-11.

2. American Psychiatric Association: Diagnostic and Statistical Manual of Mental Disorders, Fifth edition. Arlington, VA, American Psychiatric Association, 2013.

3. World Health Organization: Measuring health and disability: manual for WHO Assessment Schedule (WHODAS 2.0), Geneva, 2010.

4. http://www. who.int/classifications/icf/whodasii/en/

5. Lewis-Fernández R, Krishan Aggarwal N. Culture and psychiatric diagnosis. Adv Psychosom Med. 2013;33:15-30.

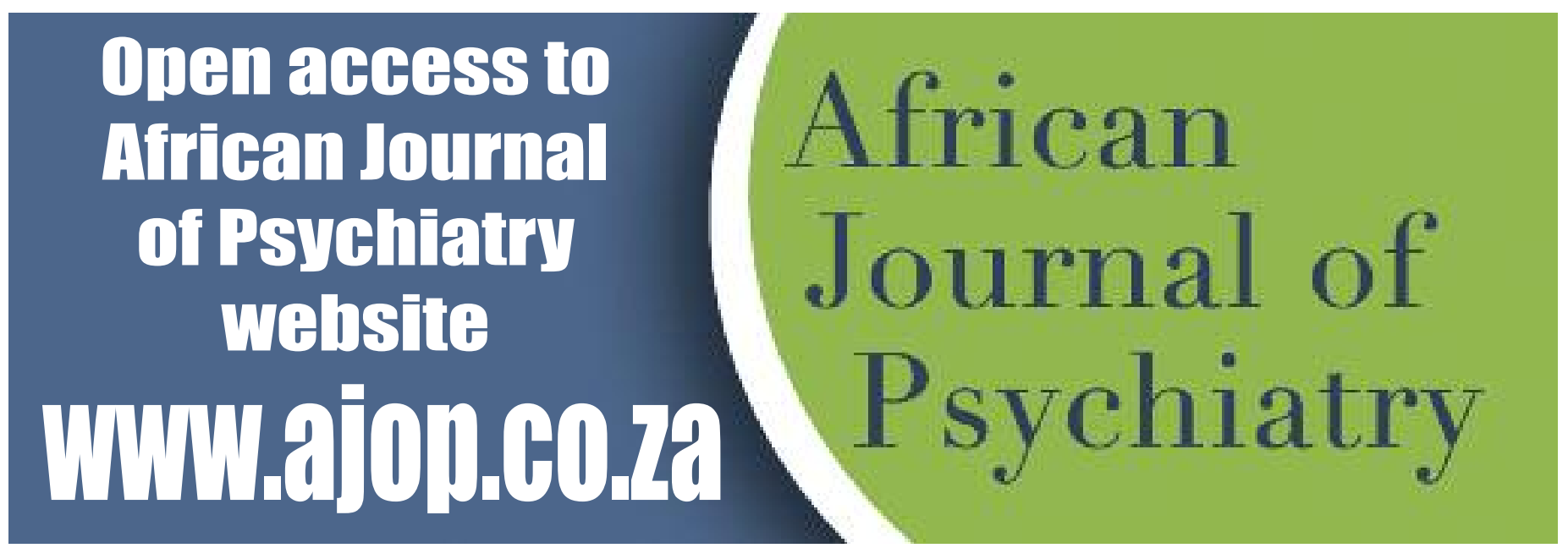

